\title{
Redução da mortalidade materna e atuação do enfermeiro
}

\author{
Reduction of maternal mortality and the nurse's acting
}

\section{Élida de Souza Barreto', Juliana de Souza Oliveira², Anne Jacob de Souza Araújo', Paula Elis de Souza Queiroz', Renata da Silva Schulz}

\author{
'Centro Universitário Jorge Amado. Salvador, Bahia, Brasil. \\ elidabarreto12@hotmail.com, annejacob@hotmail.com, paulaelis_@hotmail.com, renata.s.schulz@gmail.com \\ ${ }^{2}$ Autora para correspondência. Centro Universitário Jorge Amado. Salvador, Bahia, Brasil. juliana.soliveir@gmail.com
}

RESUMO I INTRODUÇÃO: De acordo com Sistema de Informações sobre Mortalidade (SIM), no Brasil entre 20042014 houveram 18.364 óbitos maternos. Com isso, o Ministério da Saúde (MS) tem adotado, ao longo dos anos, políticas que visam à melhoria da saúde da mulher. OBJETIVO: Avaliar os índices de mortalidade materna em Salvador e na Bahia e descrever como o enfermeiro pode atuar na redução desses índices. METODOLOGIA: Trata-se de uma pesquisa ecológica, descritiva de abordagem quantitativa, com informações coletadas sobre a mortalidade materna em Salvador e Bahia nos últimos dez anos, por meio do DATASUS. RESULTADOS: Na Bahia, segundo os casos notificados de mortalidade materna foram registrados 1.764 óbitos maternos nos anos de 2004 a 2014 . Em Salvador o ano de 2004 apresentou o menor índice de mortalidade materna com 17 óbitos, podendo estar associado à expansão do PSF, principal meio de atuação do enfermeiro para redução da mortalidade através do programa de pré-natal. Já em 2008 apresentou um somatório de 36 óbitos notificados, esses podem ter relação com a baixa cobertura populacional do programa e problemas estruturais e operacionais do serviço nos últimos anos. CONCLUSÃO: $O$ índice de óbitos maternos ainda se mostra elevado na Bahia e em Salvador. Contudo, verificou-se a importância da atuação do enfermeiro para a redução dessas taxas, pois a prevenção se dá na atenção básica, ambiente onde o enfermeiro possui autonomia regulamentada em lei para prestar o cuidado pré-natal qualificado.

PALAVRAS-CHAVE: Mortalidade materna. Assistência pré-natal. Saúde da mulher. Enfermagem

\begin{abstract}
INTRODUCTION: According to the Sistema de Informação sobre Mortalidade (SIM), in Brazil between 2004-2014 there were 18.364 maternal deaths. Thus, the Ministry of Health (MS) has adopted, over the years, policies aimed at improving women health. OBJECTIVE: To evaluate the maternal mortality rates in Salvador and Bahia and describe how the nurse can act in the reduction of these indices. METHODOLOGY: This is an ecological, descriptive, quantitative approach, with information collected on maternal mortality in Salvador and Bahia in the last ten years, through DATASUS. RESULTS: In Bahia, according to reported cases of maternal mortality, 1,764 maternal deaths were registered in 2004 to 2014. In Salvador, 2004 had the lowest maternal mortality rate with 17 deaths, may be associated with the expansion of PSF, the nurse's main means of acting to reduce mortality through prenatal program care. In 2008, there were 36 reported deaths, which may be related to the low population coverage of the program and structural and operational problems of the service in recent years. CONCLUSION: The rate of maternal deaths is still high in Bahia and Salvador. However, it was verified the importance of the nurse's role in reducing these rates, since prevention occurs in primary care, an environment where nurses have autonomy regulated by law to provide qualified prenatal care.
\end{abstract}

KEYWORDS: Maternal mortality. Prenatal assistance. Women's health. Nursing. 


\section{Introdução}

A Organização Mundial de Saúde - OMS caracteriza mortalidade materna como a morte de uma mulher no período gestacional ou puerperal, sendo este compreendido 42 dias após o término da gestação, independentemente da duração ou localização da gravidez, podendo ser relacionada a qualquer causa, seja essa agravada pela situação gestacional ou através de intervenções prestadas a ela, exceto as causas acidentais ou incidentais'.

De acordo com o Guia de Vigilância Epidemiológica do Óbito Materno a redução da razão de mortalidade materna no mundo e, especialmente no Brasil, ainda é um enorme desafio para a saúde e sociedade. Apesar dos avanços na diminuição das taxas, no ano de 2015 ainda foi observado altos índices de mortalidade por causas evitáveis, configurando assim como um grave problema de saúde pública que atinge de diferentes formas as regiões brasileiras ${ }^{2}$.

Segundo o Sistema de Informações sobre Mortalidade (SIM), no Brasil entre 2004-2014 houve 18.364 óbitos maternos, sendo a região Nordeste a mais acometida, com quantitativo de 6.514 óbitos, equivalente a $35,47 \%$ da mortalidade materna do país, e a região Centro-Oeste com a menor taxa nacional de $7,74 \%$ (total de 1.422 óbitos confirmados e notificados) $)^{3}$.

Diante dessa realidade, o Brasil juntamente a 191 outros países, em 2000, assinaram um pacto para reduzir a desigualdade e melhorar o Índice de Desenvolvimento Humano (IDH) no mundo até o período de 2015. Neste documento foram fixadas oito metas chamadas Objetivos de Desenvolvimento do Milênio (ODM), e dentre elas destacamos a quinta que traz como objetivo a redução da mortalidade materna com uma meta estipulada de 35 óbitos por 100.000 nascidos vivos (NV) 4 .

Desde que foi firmado esse pacto, observa-se no Brasil uma redução significativa na taxa de mortalidade materna, porém ainda insuficiente para alcançar a meta estipulada. Segundo o Relatório Nacional de Acompanhamento de Objetivos de Desenvolvimento do Milênio no ano de 2011 a taxa estagnou-se para menos de 64 óbitos por 100 mil NV. Vale ressaltar que dentre as principais causas de mortalidade materna estão as obstétricas diretas, relacionadas às complicações no período gestacional, parto ou puerpério, por omissão, intervenções e tratamentos incorretos, como: hipertensão, hemorragias, abortos e infecções puerperal ${ }^{5}$.

Nesse contexto o Ministério da Saúde tem adotado, ao longo dos anos, políticas que visem à melhoria da saúde da mulher. Dentre algumas pode-se destacar - Projeto Maternidade Segura de 1996; o Programa de Humanização do Pré-Natal e Nascimento (PHPN) de 2000; ○ Pacto Nacional pela Redução da Mortalidade Materna e Neonatal de 2002; A Política Nacional de Atenção Integral a Saúde da Mulher (PAISM) de 2003; A Lei do Acompanhante de 2005; e o destaque para a Rede Cegonha de 2011 . Esses programas e políticas trazem à tona a necessidade de que seja considerada assistência à saúde da mulher em todo o seu período de vida ${ }^{6}$, e trazem o enfermeiro como principal aliado na prevenção e redução da mortalidade materna.

Para isso o Ministério da Saúde tem reconhecido a importância da assistência de enfermagem na obstetrícia e inserido estes profissionais, cada vez com mais autonomia, nos programas e portarias que tratam da temática, objetivando agir na redução dos índices supracitados e garantir os direitos da mulher à vida e saúde ${ }^{7}$.

A redução das taxas de mortalidade materna no Brasil está intimamente associada à qualidade da assistência prestada às gestantes e ao acesso destas aos serviços de saúde. Neste sentido, a motivação para realização dessa pesquisa deu-se pela grande relevância na saúde pública, visto que o Brasil não conseguiu atingir a meta estipulada pela OMS, e também pela importância da prática assistencial do profissional enfermeiro na identificação precoce dos principais agravos relacionados à mortalidade materna.

Diante do que foi exposto elaborou-se as seguintes questões norteadoras: como estão os índices de mortalidade materna na cidade de Salvador e no estado da Bahia? Como o enfermeiro pode atuar para reduzir a mortalidade materna? Sendo assim, este artigo tem como objetivos: avaliar os índices de mortalidade materna em Salvador e na Bahia e descrever como o enfermeiro pode atuar de forma a diminuir esses índices. 


\section{Metodologia}

Realizou-se uma pesquisa ecológica, a qual compara-se a ocorrência de uma condição/doença relacionada à saúde e a exposição da coletividade, sem abordar informações sobre essa, mas trazendo dados sobre o grupo, a fim de verificar possível relação de comparação entre elas ${ }^{8}$, e descritiva de abordagem quantitativa, com informações coletadas a respeito da mortalidade materna em Salvador nos últimos dez anos, por meio do Departamento de Informática do Sistema Único de Saúde (DATASUS) do Ministério da Saúde.

Os dados foram coletados no site: http://www2. datasus.gov.br/DATASUS/, utilizando as informações do período de 2004 a 2014, uma vez que estas estão disponíveis apenas até o ano de 2014. Os dados foram organizados mediante tabelas elaboradas no programa Word do Microsoft Office, e discutidos a partir de referenciais teóricos que contemplem a temática abordada.

Para embasar a discussão sobre como o enfermeiro pode atuar de forma a diminuir os índices identificados, foi realizada uma pesquisa nas bases de dados: SCIELO (ScientificElectronic Library Online) e LILACS (Literatura Latino-Americana e do Caribe em Ciências da Saúde), utilizando os seguintes descritores em Ciências da Saúde (Decs): mortalidade materna, assistência pré-natal, saúde da mulher, e enfermagem.

Para seleção dos artigos utilizamos como critérios de inclusão: artigos em português, disponíveis na íntegra e entre os anos de 2006 à 2016, e, como critério de exclusão: artigos fora do período estabelecido e que não contemplam a temática. Após passar pelos critérios estabelecidos restaram 20 artigos aos quais foram lidos os títulos e resumos de forma minuciosa para identificar os que atendiam ao objetivo da pesquisa, selecionando os que condiziam com o tema do trabalho, restando assim 17 artigos lidos. Foram excluídos os repetidos e in- completos, contemplando assim 07 artigos que se fizeram apropriados para embasar a discussão dos resultados desta pesquisa, bem como manuais e relatórios do Ministério da Saúde.

Por se tratar de um banco de domínio público, não foi necessário submeter o projeto ao Comitê de Ética em Pesquisa, visto que não envolve diretamente seres vivos, o que está de acordo com a resolução $466 / 2012$, que norteia as pesquisas com seres humanos?.

\section{Resultados e discussão}

Na Bahia, frente às informações disponíveis no DATASUS, foram registrados 1.764 casos notificados de óbitos maternos entre os anos de 2004 a 2014. O estado é considerado como tendo um alto indicador de mortalidade materna desde 1990, e apesar de declínios existentes ao passar dos anos, o índice ainda é elevado uma vez que os óbitos maternos estão associados à raça negra, predominante na população baiana, sendo tal raça predisposta a complicações hemorrágicas e Doenças Hipertensivas Específicas da Gestação (DHEG), portanto um aumento dessa população aumenta também o risco de óbito materno ${ }^{10}$.

Em relação ao quantitativo de casos apresentados em Salvador, a tabela 1 mostra, em comparação aos dados da Bahia, que os principais anos de maior e menor notificação se diferem. Em Salvador, no ano de 2008 houve o maior índice de mortalidade materna. Vale salientar que nesse mesmo ano, na Bahia, ocorreu um quantitativo elevado de óbitos, somando 172 óbitos $(9,75 \%$ do total de óbitos do estado). A Bahia em 2007 apresentou a menor taxa, com 146 óbitos maternos confirmados e notificados, correspondendo a $8,27 \%$ do total de óbitos do estado. Em 2009 apresentou a maior taxa com 195 óbitos notificados, cerca de $11,05 \%$ do total de casos do Estado no período de 2004 a 2014.

Tabela 1. Óbito materno por ano segundo estado (Bahia) no período de 2004-2014.

\begin{tabular}{lllllllllllll}
\hline Estado & 2004 & 2005 & 2006 & 2007 & 2008 & 2009 & 2010 & 2011 & 2012 & 2013 & 2014 & TOTAL \\
\hline Bahia & 161 & 164 & 158 & 146 & 172 & 195 & 161 & 149 & 147 & 162 & 149 & 1.764 \\
\hline
\end{tabular}

Fonte: MS/SVS/CGIAE - SIM 
Visto que Salvador é o maior município do Estado, a tabela 2 apresenta o total de casos notificados de óbitos maternos entre os anos de 2004 a 2014, com um total de 282 mortes.

Tabela 2. Óbito materno por ano segundo município (Salvador) no período de 2004-2014.

\begin{tabular}{lllllllllllll}
\hline Município & 2004 & 2005 & 2006 & 2007 & 2008 & 2009 & 2010 & 2011 & 2012 & 2013 & 2014 & TOTAL \\
\hline Salvador & 17 & 18 & 26 & 21 & 36 & 32 & 30 & 21 & 24 & 24 & 33 & 282 \\
\hline
\end{tabular}

Fonte: MS/SVS/CGIAE - SIM

De acordo com a tabela 2 podemos observar que em 2004 houve a menor taxa, 6,03\% com um total de 17 óbitos notificados. Já em 2008 ocorreu um crescimento do número de mortalidade, com 36 óbitos, equivalente a $12,76 \%$ do total do município. Esse aumento pode estar associado à regulamentação da portaria $\mathrm{n}^{\circ} 1.172 / 2004$ que define o serviço de vigilância epidemiológica como atribuição dos municípios e estados ${ }^{10}$.

Esses valores elevados podem estar associados à maior notificação de óbitos ou a vigilância mais organizada, assim como, os menores valores devem ser analisados com cautela, pois podem ser resultado de medidas de controle e prevenção da mortalidade, bem como subnotificação dos óbitos maternos.

Segundo a OMS, em 2015 houve queda de 43\% nos números de mortalidade global quando comparados a década de 90 , estimando-se uma redução de 532.000 mortes em 1990, para 303.000 óbitos maternos em 2015. Nesses últimos 25 anos, período entre 1990 e 2015 , ocorreu uma redução de $44 \%$ no índice de mortalidade materna no mundo, equivalente a diminuição de 385 óbitos maternos por 100.00 NV em 1990, para 216 óbitos maternos por $100.00 \mathrm{NV}$ em $2015^{11}$.

Apesar da acentuada queda no número de óbitos maternos, nenhum país conseguiu alcançar as metas estipuladas em 2015. Isso permitiu que fossem acordados novos ODM e novas metas no período de 2016 à 2030, com o intuito de reduzir a taxa global da mortalidade materna para 70 mortes por $100.00 \mathrm{NV}$ e no Brasil para 20 mortes por $100.00 \mathrm{NV}^{12}$.

No Brasil, em 2014, o total de óbitos maternos foi de 1.739 casos notificados ${ }^{3}$. Em 2015 esse dado apresentou um decréscimo totalizando 1.300 mor- tes, equivalente a taxa de 44 para $100.00 \mathrm{NV}^{12}$. Essa redução ao longo dos anos é resultado da vigilância dos Comitês de Mortalidade Materna, Estadual e Municipal, criados pelo MS em 1987, que, através de estratégias e acompanhamento das políticas públicas em saúde da mulher, propõem medidas de melhoria das informações e registro dos óbitos, utilizando-se destas para promover ações que contribuam para a redução das taxas de mortalidade ${ }^{2}$.

É importante ressaltar que dois fatores dificultam o real acompanhamento dessas taxas: a sub-informação das declarações de óbito, resultante do preenchimento errado e omissão das causas de morte relacionada à gestação, assim como, o sub-registro, fruto da omissão do registro do óbito nos cartórios, comum nas regiões norte e nordeste, devido a condições socioeconômicas e culturais ${ }^{13}$.

Pensando nisso o governo da Bahia, em 2007, através do decreto $n^{\circ} 10.263$ incluiu as ocorrências de óbitos maternos na lista de notificação compulsória e investigação imediata, a fim de proporcionar um perfil real desse indicador no Estado e atuar de forma preventiva para sua redução ${ }^{14}$.

Com o avanço do programa de agentes comunitários de saúde (PACS) e o Programa Saúde da Família (PSF), observou-se redução dos números de mortalidade materna, conforme visto na tabela 1 . Isso porque entre o período de expansão do PSF, aproximadamente entre 2003 e 2007, houve uma reorganização da atenção básica, ampliando a cobertura de atenção à saúde da população e consequentemente maior acesso ao pré-natal.

Apesar das medidas implantadas para redução da mortalidade materna, observa-se a partir de 2008, na Bahia e em Salvador, acentuada dispari- 
dade nos óbitos maternos, uma vez que, segundo o Departamento de Atenção Básica (DAB), em 2014 o percentual de cobertura do PSF na capital foi de $26,34 \%$, totalizando em 714.150 a cobertura populacional das equipes de saúde, sendo esta considerada baixa em relação a população total de 2.710.968 habitantes. Essa disparidade pode estar associada a problemas encontrados no PSF nos últimos anos, como: a baixa cobertura do acompanhamento de planejamento familiar; baixa cobertura e diminuição da assistência pré-natal, levando a falhas na identificação de comorbidades de risco a saúde da mulher e do bebê, e tratamento destas durante a gestação; além da fragilidade na busca ativa de gestantes; identificação das gestações de risco bem como referência e acesso aos serviços de maior complexidade ${ }^{15}$.

Dessa forma a assistência pré-natal é uma importante aliada na redução da mortalidade materna, implantada pelo Ministério da Saúde em 2000 no Programa de Humanização do Pré-Natal e Nascimento (PHPN), sendo recomendado que em média haja a realização de seis consultas de pré-natal, iniciadas desde o primeiro trimestre da gravidez, para a detecção precoce de riscos e complicações evitáveis; uma consulta no período do puerpério, essa até 42 dias após o parto; e a recomendação durante o pré-natal da realização dos exames laboratoriais juntamente às orientações a respeito do aleitamento materno ${ }^{16}$.

No intuito de reduzir a mortalidade materna foram implantados programas que possivelmente influenciaram nos óbitos maternos apresentados, como por exemplo, no ano de 2011, na Bahia e em Salvador, onde $\circ$ índice de mortalidade materna foi menor quando comparado aos demais anos. Vale destacar que em 2011, para reforçar a atenção a saúde da mulher, implantou-se a Rede Cegonha que tem como objetivos: um novo modelo de atenção ao parto, nascimento e à saúde da mulher e da criança; garantia de acesso, acolhimento e resolutividade, e redução da mortalidade materna e neonatal; e como diretrizes a garantia das boas práticas e segurança na atenção ao parto e nascimento, e melhoria da qualidade do pré-natal ${ }^{17}$.

Sendo assim, percebe-se que a implantação da Rede Cegonha possibilitou ao enfermeiro um importante papel nesse processo, uma vez que a rede permite maior autonomia e subsídios necessários para a atuação nos serviços de pré-natal, puerpério e atenção à saúde da mulher e da criança. Aliado a isso proporciona capacitação para promover assistência qualificada capaz de contribuir para redução da mortalidade materna.

Segundo o Decreto $n^{\circ} 94.406 / 8724$, a Lei de Exercício Profissional da Enfermagem dá cobertura ao enfermeiro para realizar consultas de pré-natal de baixo risco, pois possui conhecimento teórico-científico para prestar a assistência ${ }^{18,19}$.

Neste contexto, a atenção ao pré-natal é fundamental quando se trata de redução dos índices de mortalidade materna e perinatal. Esse serviço, de acordo com o MS é de fácil acesso e contempla os seguintes níveis de atenção: promoção, prevenção e assistência as gestantes e recém-nascidos ${ }^{20}$, sendo o enfermeiro o profissional mais atuante no pré-natal, - qual é munido de protocolos de atribuição profissional que os auxiliam a prestarem uma assistência de qualidade ${ }^{21}$.

Essa redução da mortalidade materna se dá através de medidas para eliminação dos casos de doenças inerentes à gestação e perpassa a assistência hospitalar com o incentivo ao parto normal visando a redução das cesáreas desnecessárias e consequentemente $\circ$ óbito materno ${ }^{22}$. Além disso, devem ser desenvolvidas ações no período pós parto, onde se torna indispensável à atenção a mulher e ao recém-nascido por meio da consulta de puerpério. Logo deve ser incentivado durante o acompanhamento pré-natal como também na maternidade, 0 retorno à UBS após o parto ${ }^{23}$.

Na atenção básica, o enfermeiro é o principal responsável por essas medidas de eliminação dos riscos pertinentes à gravidez, parto e puerpério. Nesse sentindo durante o pré-natal este profissional deve garantir o acolhimento e acompanhamento necessário para que a gestante possa conduzir de maneira saudável sua gestação ${ }^{24}$. Entretanto, este acompanhamento também é imprescindível para a busca ativa das gestantes que não iniciaram ou abandonaram as consultas,

Portanto, cabe ao enfermeiro, no cuidado pré-natal, a necessidade de criar estratégias para as gestantes a fim de que essas compareçam nas próximas 
consultas para continuidade da assistência. É de grande importância ir além das consultas marcadas, utilizando como estratégia de alcance os grupos de gestantes com ações educativas sobre o período gestacional, processo de vinculação a maternidade, importância da consulta puerperal, saúde bucal do binômio mãe e filho, e explicar sobre as particularidades do aleitamento materno e do planejamento familiar, bem como temáticas sugeridas por elas.

Sendo assim, para uma assistência qualificada é imprescindível que haja a capacitação do profissional de enfermagem, para que se tornem aptos a prestarem assistência digna à mulher ao longo do ciclo gravídico-puerperal, podendo essa capacitação ser complementada por uma especialização em enfermagem obstétrica ${ }^{19}$.

A implantação dos Centros de Parto Normal proporcionou ao enfermeiro obstetra a capacidade de intervir em ações obstétricas desnecessárias, gerando sentimento satisfatório às parturientes, através da prestação da assistência humanizada e tratando a parturiente em seus aspectos psicossociais, o que por sua vez articula meios que podem reduzir o risco de mortalidade materno-infantil ${ }^{25}$.

Nesse contexto, os altos índices de óbitos maternos apresentados neste estudo apontam a necessidade na redução dessas taxas de mortalidade, já que estas são um importante indicador da qualidade da assistência materno-infantil, tendo como principal aliado o enfermeiro, o qual promove medidas que visam a redução da mortalidade por meio de ações de promoção a saúde materna e prevenção de riscos inerentes a gestação. Tais ações estão embasadas por protocolos e manuais técnicos instituídos pelo Ministério da Saúde que reconhece o enfermeiro como peça fundamental no progresso para redução do perfil da mortalidade materna ${ }^{7}$.

\section{Considerações finais}

Durante o período de 1990 a 2015 o mundo concentrava esforços para diminuir o índice global de mortalidade materna. Nesse contexto o Brasil a fim de atingir a meta estipulada no $5^{\circ} \mathrm{ODM}$ implantou uma série de políticas e programas para melhoria da assistência à saúde da mulher e consequentemente redução das taxas de óbitos maternos.

Frente aos objetivos de avaliar os índices de mortalidade materna na Bahia e em Salvador e atuação do enfermeiro para redução dessas taxas, os resultados dessa pesquisa apontaram que mesmo com o declínio dos óbitos maternos registrados e com as implantações das políticas, o índice ainda se mostra elevado na Bahia e em Salvador. Isso pode estar relacionado ao aumento da vigilância de óbitos, bem como aumento da população predisposta aos riscos inerentes a gravidez, além da fragilidade na cobertura de saúde da população pela ESF.

Verificou-se a importância da atuação do enfermeiro para a redução dessas taxas, pois a prevenção se dá principalmente na atenção básica, ambiente onde o enfermeiro possui autonomia regulamentada em lei para prestar o cuidado pré-natal qualificado, estando atento para garantia da continuidade da assistência e com isso identificação de comorbidades que possam vir a colocar em risco a saúde da mulher.

Vale ressaltar que foram encontrados poucos estudos referente a mortalidade materna na Bahia e em Salvador, o que reforça a importância de uma maior produção científica voltada para esta temática.

\section{Conflitos de interesses}

Nenhum conflito financeiro, legal ou político envolvendo terceiros (governo, empresas e fundações privadas, etc.) foi declarado para nenhum aspecto do trabalho submetido (incluindo mas não limitandose a subvenções e financiamentos, conselho consultivo, desenho de estudo, preparação de manuscrito, análise estatística, etc).

\section{Referências}

1. Organização Mundial de Saúde. Classificação Estatística Internacional de Doenças e Problemas Relacionados à Saúde. 10.ed. São Paulo: CBCD; 1995.

2. Ministério da Saúde (BR). Secretaria de Atenção à Saúde. Manual dos comitês de mortalidade materna. Brasília: Ministério da Saúde; 2009. 
3. DATASUS. Departamento de Informática do Sistema Único de Saúde [Internet]. [acesso em 2017 fev 02]. Disponível em: http://tabnet.datasus.gov.br/cgi/tabcgi.exe?sim/cnv/ mat10uf.def

4. Ministério da Saúde (BR). Secretaria de Vigilância em Saúde. Guia de vigilância epidemiológica do óbito materno. Brasília: Ministério da Saúde; 2009.

5. IPEA. Objetivos de desenvolvimento do milênio: relatório nacional de desempenho. Brasília: IPEA; 2014.

6. Narchi NZ, Cruz EF, Gonçalves R. O papel das obstetrizes e enfermeiras obstetras na promoção da maternidade segura no Brasil. Ciênc saúde coletiva. 2013;18(4):1059-68. doi: $10.1590 /$ S1413-81232013000400019

7. Barbastefano PS, Vargens OMC. Prevenção da mortalidade materna: desafio para o enfermeiro. Rev bras enferm. 2009;62(2):278-82. doi: 10.1590/S003471672009000200017

8. Lima-Costa MF, Barreto SM. Tipos de estudos epidemiológicos: conceitos básicos e aplicações na área do envelhecimento. Epidemiol Serv Saúde. 2003;1 2(4):189-201. doi: $10.5123 /$ S1679-49742003000400003

9. Brasil. Resolução $N^{\circ}$ 466, de 12 de dezembro de 2012. Diretrizes e normas regulamentadoras de pesquisa envolvendo seres humanos. Diário Oficial da União. 2012 dez. 12.

10. Ribeiro L. Morbimortalidade materna no estado da Bahia: diferenciais segundo a raça/cor da pele [dissertação]. Feira de Santana: Universidade Estadual de Feira de Santana; 2013.

11. World Health Organization. Trends in maternal mortality: 1990 to 2015: estimates by WHO, UNICEF, UNFPA, World Bank Group and the United Nations Population Division. Geneva: World Health Organization; 2015.

12. Souza JP. A mortalidade maternal e os novos objetivos de desenvolvimento sustentável (2016-2030). Rev Bras Ginecol Obstet. 2015;37(12):549-51.

13. Ministério da Saúde (BR). Secretaria de Atenção à Saúde. Pacto Nacional pela Redução da Mortalidade Materna. Brasília: Ministério da Saúde; 2004.

14. Secretaria da Saúde do Estado da Bahia. Superintendência de Vigilância e Proteção da Saúde. Diretoria de Informação em Saúde. Perfil da Morbimortalidade da População da Bahia [Internet]. 2010 [acesso em 2017 fev 19]. Disponível em: http:// www.suvisa.ba.gov.br/sites/default/files/documentos/ arquivo/2010/12/28/BIS_V\%201\%20Revista_ Morbimortalidade_26.05.10[1].pdf

15. Secretaria da Saúde do Estado da Bahia.

Superintendência de Vigilância e Proteção da Saúde. Análise dos Óbitos Maternos do ano de 2014 na Bahia: Câmara Técnica Estadual de Análise de Óbito [Internet]. 2016. [acesso em 2017 mar 13]. Disponível em: http://www.suvisa.ba.gov.br/sites/default/files/ vigilancia_epidemiologica/doencas_transmissiveis/ arquivo/2016/10/24/Apresenta $\%$ C3\%A7\%C3\%A30\%20 Estudo\%20\%C3\%93bitos\%20Maternos\%202014_ Semin\%C3\%A1 rio\%20DCNT_VEO2016.pdf

16. Polgliane RBS, Leal MC, Amorim MHC, Zandonade E, Santos Neto ET. Adequação do processo de assistência pré-natal segundo critérios do Programa de Humanização do Pré-natal e Nascimento e da Organização Mundial de Saúde. Ciênc Saúde Coletiva. 2014;19(7):1999-2010. doi: $10.1590 / 1413-81232014197.08622013$

17. Brasil. Portaria ${ }^{\circ} 1.459$ de 24 de junho de 2011. Institui no âmbito do Sistema Único de Saúde-SUS- a Rede Cegonha. Diário Oficial da União. 2011 jun 24 [acesso em 2017 fev 02]. Disponível em: http://bvsms.saude.gov.br/bvc/ saudelegis/gm/2011/prt1459_24_06_2011.html

18. Brasil. Decreto n 94.406 de 08 de junho de 1987. Dispõe sobre o exercício da enfermagem e dá outras providências. Diário Oficial da União. 1987 jun 08.

19. Cunha MA, Mamede MV, Dotto LMG, Mamede FV. Assistência pré-natal: competências essenciais desempenhadas por enfermeiros. Esc Anna Nery Rev Enferm. 2009;13(1):145-153. doi: 10.1590/S1414$\underline{81452009000100020}$

20. Ministério da Saúde (BR). Secretaria de Atenção à Saúde. Pré-natal e puerpério: atenção qualificada - manual técnico. Brasília: Ministério da Saúde; 2006.

21. Rodrigues EM, do Nascimento RG, Araújo A. Protocolo na assistência pré-natal: ações, facilidades e dificuldades dos enfermeiros da Estratégia de Saúde da Família. Rev Esc Enferm USP. 2011 ;45(5):1041-47. doi: 10.1590/S0080$\underline{62342011000500002}$

22. Schirmer, J. A importância da enfermeira nas ações governamentais de redução de morbi mortalidade materna e neonatal. Acta Paul Enferm. 2007;20(3):5. doi: 10.1590/ S0103-21002007000300001

23. Secretaria de Estado da Saúde de São Paulo. Manual Técnico do Pré-natal e Puerpério. São Paulo: Secretaria de Estado da Saúde de São Paulo; 2010.

24. Silva TGC, Atunes TKT, Gramacho RCCV. Razões que levam adolescentes grávidas a permanecerem nas consultas pré-natais numa maternidade pública de salvador. Rev Enferm Contemp. 2014;3(1):51-60. doi: 10.17267/23173378rec.v3i1.297

25. Oliveira EM, Celentto DD. A temática da Rede Cegonha e a inserção do enfermeiro nesse contexto. Revista de Saúde. 2016;7(1):33-38. doi: $10.21727 /$ rs.v7i1.87 